\title{
A Fast Firing Binaural System for Ultrasonic Pattern Recognition
}

\author{
Fernando Moita • Ana C. Lopes • Urbano Nunes
}

Received: 5 December 2006 / Accepted: 6 May 2007 /

Published online: 3 July 2007

(C) Springer Science + Business Media B.V. 2007

\begin{abstract}
A binaural sonar configuration with capability to detect and identify walls, edges and corners on real-time is presented in this work. A new multi-echo ultra-fast firing method increases the sonar acquisition rate, and provides crossed measurements without interference. A feature map is built on-line using Bayesian updating and classification rules. Three classifiers are implemented and analyzed: minimum risk (MR), maximum a posteriori (MAP), and minimum distance (MD). Experimental results of ultrasonic reflector recognition, using data collected in a specular indoor environment are presented in the paper.
\end{abstract}

Keywords Sensors • Sensor systems • Ultrasonic transducers • Robot navigation • Pattern identification $\cdot$ Classification $\cdot$ Bayesian classification

\section{Introduction}

Thanks to their biological sonar, bats can distinguish between objects that are just $0.3 \mathrm{~mm}$ apart, know the size, the location, the speed, the direction of the movement and even the thickness of the insect they are hunting [19]. All this, collision-free, through a cave teeming with thousands of other bats and their calls, in total darkness or under heavy rain. These amazing abilities certainly persuade the more sceptical to accept the potentialities of the ultrasonic acoustic sensors.

F. Moita · A. C. Lopes $(\varangle) \cdot$ U. Nunes ISR, Institute of Systems and Robotics, Department of Electrical and Computer Engineering, University of Coimbra, 3030-290 Coimbra, Portugal e-mail: anacris@isr.uc.pt 
Ultrasound TOF $^{1}$ sensors have been around for a while, used in mobile robot applications such as distance measurement, environment perception and robot navigation $[12,14]$. However, they have been only marginally successful. In many applications like environment perception or robot navigation, ultrasonic sensors have been considered too unreliable and inaccurate, due to the wide opening angle of this type of sensors that introduce a high angular uncertainty. This fact is due, essentially, to the inefficient use of ultrasound information.

On the other hand ultrasound sensors are simple in construction and use, mechanically robust, and they provide a cost-effective process for environment perception. The widely used Polaroid device is inexpensive, easily integrated and has found wide use in robotic applications.

Due to the importance of ultrasound sensors, much of the latest research work has been focused on the ultrasound rangefinder data interpretation and improvement [17].

Regarding data interpretation, several physical simulation models [12] and experimental based models derived from data collection $[7,14]$ have been addressed. Detection and identification of reflectors using multiple sensor arrangements has been intensively researched. One of the earliest systems, with two ultrasonic sensors [2], was only able to differentiate between walls and edges, using TOF and amplitude as source information. Afterwards, a new system with three active (movable) sensors [18], providing TOF and amplitude information, was able do classify the reflectors as small (edges) or large (walls and corners). Later on, a special combination of two transmitters and two receivers [10] using only TOF information had the ability to detect and distinguish among the three basic reflectors. A triaural system which has the ability to determine the curvature of any reflector between the edge and the wall is reported in [16]. The three basic reflectors can also be identified by a four-TOF sensor system [9]. Lastly, a simple system with only two rotative Polaroid transducers and TOF information [1] was able to detect and distinguish all the basic reflectors.

The ME-EERUF system [13] with only two transducers and TOF processing together with an active firing scheme, shows ability to detect and classify on realtime standard reflectors as edges, corners and walls. This new firing technique (MEEERUF) allows high data acquisition rates with a high level of error rejection, it is easy to implement with simple sensor configurations, providing essential information for reflector recognition.

The perception of reflector position and classification is of great importance, allowing the implementation of new methods for real-time map building, and can be an important source of information for robot localization, obstacle avoidance, safe navigation and environment representation $[3,6,20]$.

\section{Polaroid Sonar Sensor}

The Polaroid electrostatic transducer can transmit and receive the ultrasound waves ( $\mathrm{T} / \mathrm{R}$ transducer). Initially the transducer works as a transmitter, emitting a train of

\footnotetext{
${ }^{1}$ Time-Of-Flight.

悬 Springer
} 
Fig. 1 Polaroid 600 series transducer, radiation amplitude pattern

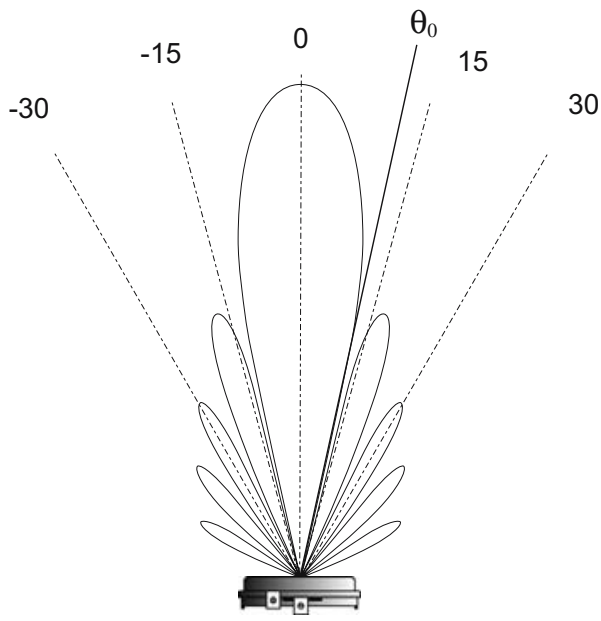

pulses and then changes to the receiver mode to detect the echoes of the reflected ultrasound wave. This type of transmitter is usually modelled by a circular piston of radius $a$, vibrating at frequency $f$. The radiated sound pressure pattern, can be modelled by equation

$$
P(\theta)=\left|\frac{2 J_{1}(k \cdot a \cdot \sin \theta)}{k \cdot a \cdot \sin \theta}\right|
$$

where $J_{1}$ is a Bessel function of the first kind first order, $k=\frac{2 \pi}{\lambda}$ where $\lambda$ is the sound wave length, $a$ is the transducer radius, and $\theta$ is the sensor orientation. A graphical representation of the pattern is depicted in Fig. 1. The information proceeding from

Fig. 2 Polaroid 600 series transducer experimental data, showing clearly the main perception areas of the sensor

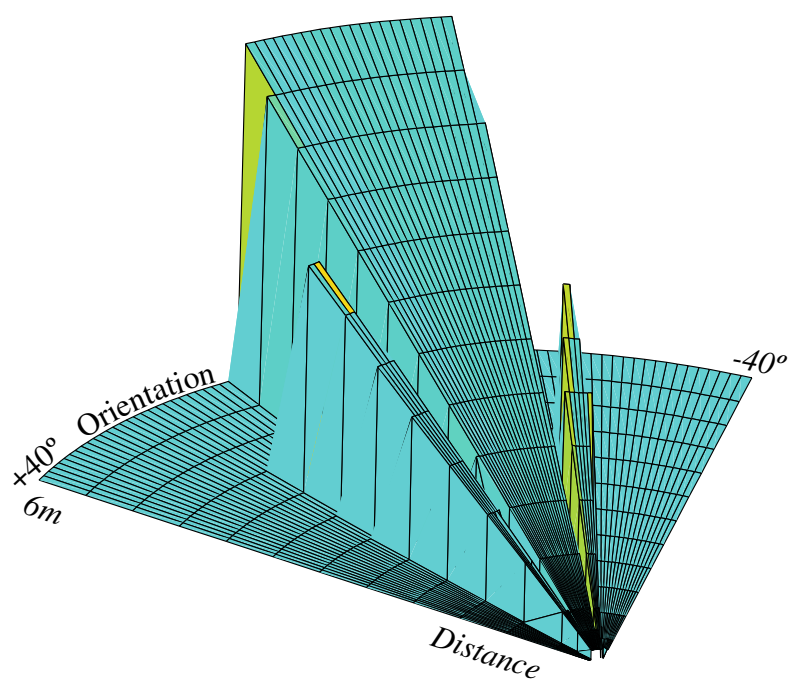


Fig. 3 Bias error $E_{S}(\theta)$ and precision $\sigma_{r}(\theta)$. The bias error of the main lobe is characterized with good precision $\left[\right.$ low $\left.\sigma_{r}(\theta)\right]$

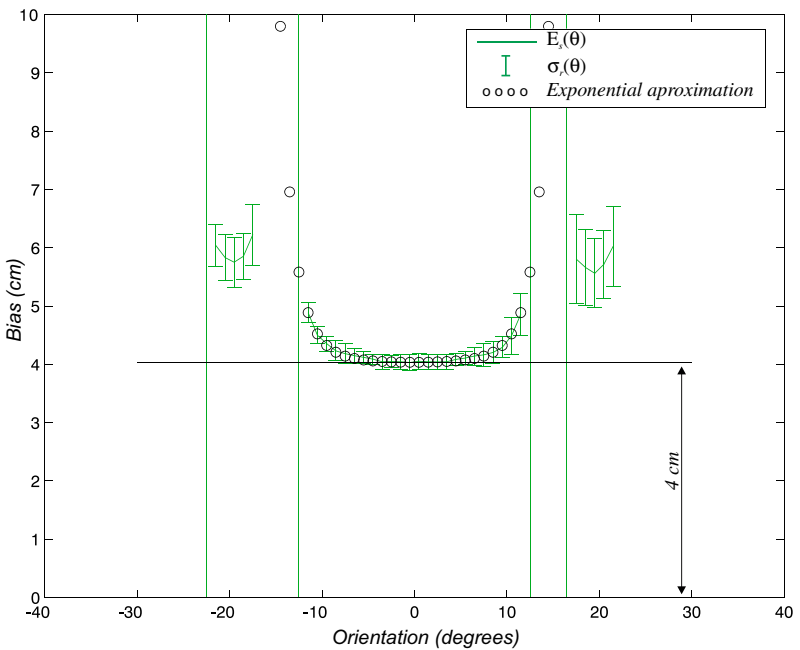

an ultrasound sensor has an inherent uncertainty due to factors like poor directivity, frequent misreading and corruption of data by interference. It is very important to understand its real behavior and develop models taking into account these sonar uncertainties.

\subsection{Experimental Sensor Modelling}

An experimental sensor modelling was carried out in a previous work [14], taking measurements in the linear range of 0.12 to $6 \mathrm{~m}$ and in the angular range of -40 to $40^{\circ}$. The orientation uncertainty is the main source of errors. In many applications, ultrasonic sensors are used as ray tracers, however experimental results reveal a large orientation uncertainty, as shown in Fig. 2. It is noticeable the main perception

Fig. 4 Linearized boundaries of perception

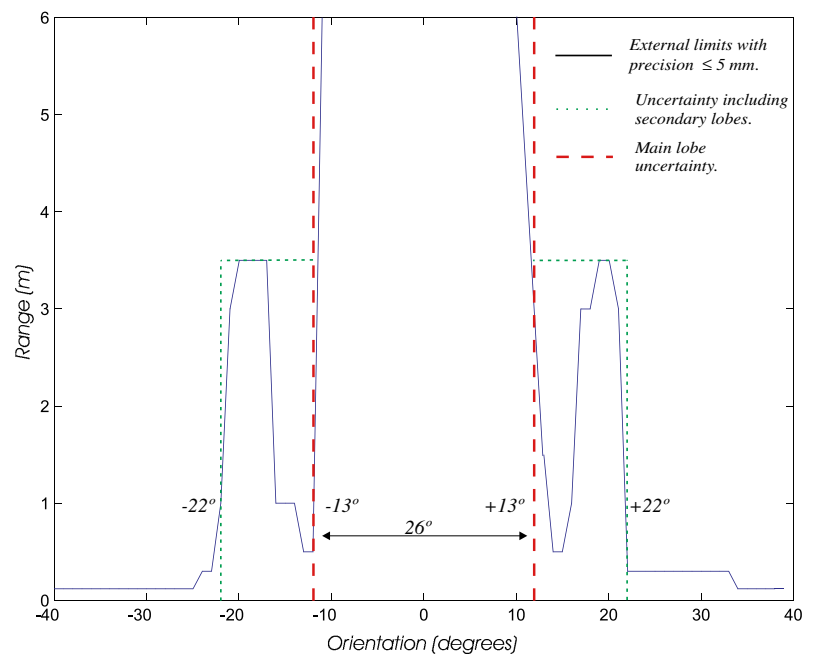


Fig. 5 Statistical perception model

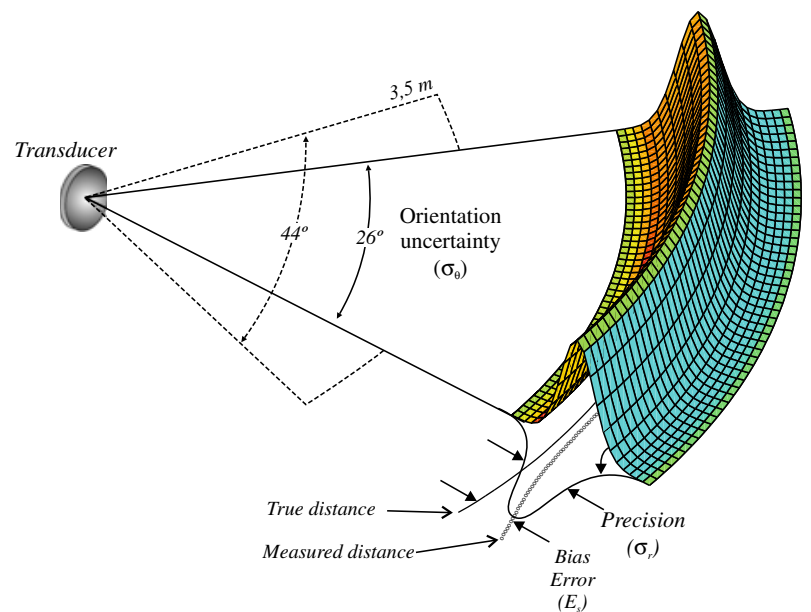

lobe $\left(-13\right.$ to $\left.13^{\circ}\right)$ and the side areas $\left(-17\right.$ to $-22^{\circ}$ and +17 to $\left.+22^{\circ}\right)$, with a short perceptual range, due to secondary lobes. The bias and precision errors, are depicted in Fig. 3. Two components make up the bias: the linear one, resulting from constant internal delays, and the exponential component, that is related to the transmitted amplitudes and perception variation with orientation. All these results are synthesized by Figs. 4 and 5, and are modelled by the following equations:

Bias for the main lobe:

$$
E_{S}(\theta)=\frac{1}{2.7 e^{\left(\frac{-4 \theta^{2}}{12.9^{2}}\right)}}+40(\mathrm{~mm})
$$

Precision:

$$
\sigma_{r}= \begin{cases}2 \mathrm{~mm} & \text { main lobe } \\ 5 \mathrm{~mm} & \text { sidelobes }\end{cases}
$$

Orientation uncertainty including side lobes:

$$
\sigma_{\theta}= \begin{cases}44^{o} & \forall r \leq 3,5 m \\ 26^{\circ} & \forall r>3,5 m\end{cases}
$$

Orientation uncertainty in main lobe:

$$
\sigma_{\theta}=26^{o} \quad \forall r
$$

\section{The ME-EERUF System}

The "error eliminating rapid ultrasonic firing (EERUF)" method [4] is the basis for the new fast firing method. EERUF allows multiple sensors to be fired faster than in conventional applications, reducing the number of misreadings. Taking into account the advantages of this method, a new functionality was introduced: the multi-echo reading. The new "multi-echo EERUF (ME-EERUF)" method has the capability 
of eliminating the undesirable echoes (resulting from indirect path crosstalk), and to identify the echoes proceeding from adjacent sensors, a "desired crosstalk" necessary to implement feature recognition algorithms.

\subsection{System Configuration and Geometries}

Often, due to design limitations, it is not practical to provide a robot with moving or complex sensor distributions to achieve optimal performance in data acquisition. Most commercial robots have settled sensor configurations, usually with rectangular distributions [10, 14]. Since many commercial mobile robots and wheelchairs are equipped with ultrasonic sensors, a sonar configuration that can be used with advantages on these types of systems is presented. The configuration depicted in Fig. 6 can be the result from any two collinear and adjacent sensors in a robot. Considering a specular environment and the virtual image concept, the geometrical configurations for the three reflectors (wall, edge and corner), are derived. From the vector of measurements $x=\left[R_{0}, R_{1}, R_{2}\right]$ acquired by the binaural system, the orientation $\left(\alpha_{W}, \alpha_{E}, \alpha_{C}\right)$ for each feature can be computed and these measurements can be related according to the following equations

Wall geometry (Fig. 6a):

$$
\begin{aligned}
R_{2 W} & =\sqrt{R_{0} R_{1}+\left(\frac{d}{2}\right)^{2}} \\
\alpha_{W} & =\cos ^{-1} \frac{R_{0}-R_{1}}{d}
\end{aligned}
$$

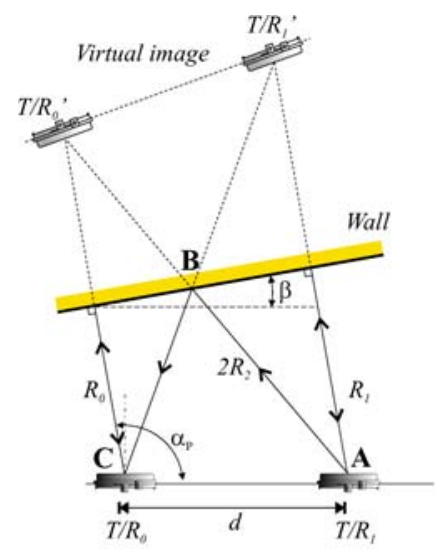

a

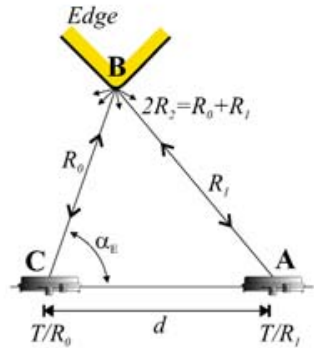

b

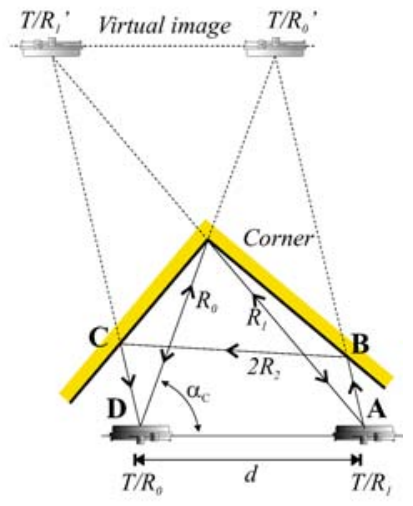

C

Fig. 6 a Virtual image geometry for the wall reflection; b diffuse edge reflection; c virtual image geometry for the corner reflection; for all reflectors, $R_{0}$ and $R_{1}$ represent the shortest distance from the respective sensors to the reflector. $R_{2}$ is half the path length, which is defined as follows: $A B C$ represents the path for the wall and for the edge, and $A B C D$ represents the path for the corner 


\section{Cycle A Cycle B}

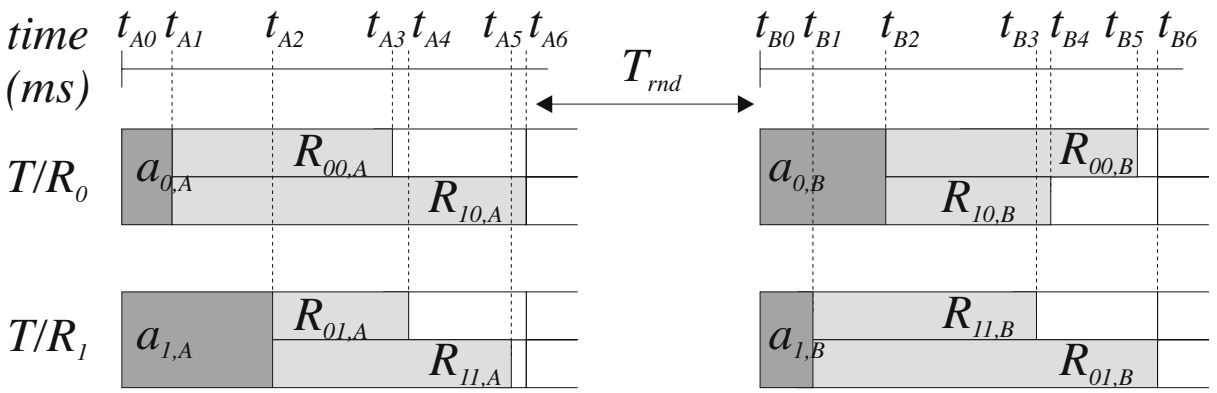

Fig. 7 Firing sequence for the ME-EERUF method, where $T_{r n d}$ represents a random time between cycles

Edge geometry (Fig. 6b):

$$
\begin{aligned}
R_{2 E} & =\frac{R_{0}+R_{1}}{2} \\
\alpha_{E} & =\cos ^{-1}\left(\frac{R_{0}^{2}-R_{1}^{2}+d^{2}}{2 d R_{0}}\right)
\end{aligned}
$$

Corner geometry (Fig. 6c):

$$
\begin{aligned}
R_{2 C} & =\sqrt{\frac{R_{0}^{2}}{2}+\frac{R_{1}^{2}}{2}-\left(\frac{d}{2}\right)^{2}} \\
\alpha_{C} & =\cos ^{-1}\left(\frac{R_{0}^{2}-R_{1}^{2}+d^{2}}{2 d R_{0}}\right)
\end{aligned}
$$

\subsection{Multi-Echo Firing Sequence}

Firing two adjacent T/R sensors at the same time, or within a short time interval, results in undesirable crosstalk. Firing two sensors with a short controlled delay allows the identification of crossed echoes from side sensors. Taking the wall reflection geometry as an example, the multi-echo firing procedure can be explained

Table 1 Notation for the firing procedure

\footnotetext{
$T / R_{k} \quad$ Sensor transmiter/receiver $k, k=\{0,1\}$

$C_{x} \quad$ Firing cycle $x, x=\{A, B\}$

$a_{k, x} \quad$ Sensor $k$ firing delay at cycle $x$

$T_{\text {rnd }} \quad$ Random delay between cycles

$R_{k j, x} \quad$ TOF of the wave emitted by sensor $k$ and received by sensor $j$, at cycle $x, j=\{0,1\}$
} 
Table 2 Decision rules for the ME-EERUF system

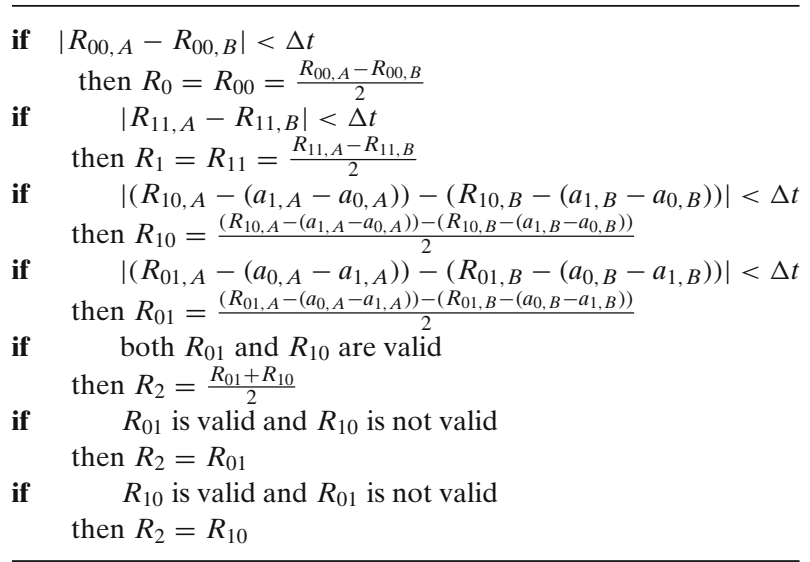

by the firing sequence of Fig. 7, and the following step sequence, with notations presented in Table 1.

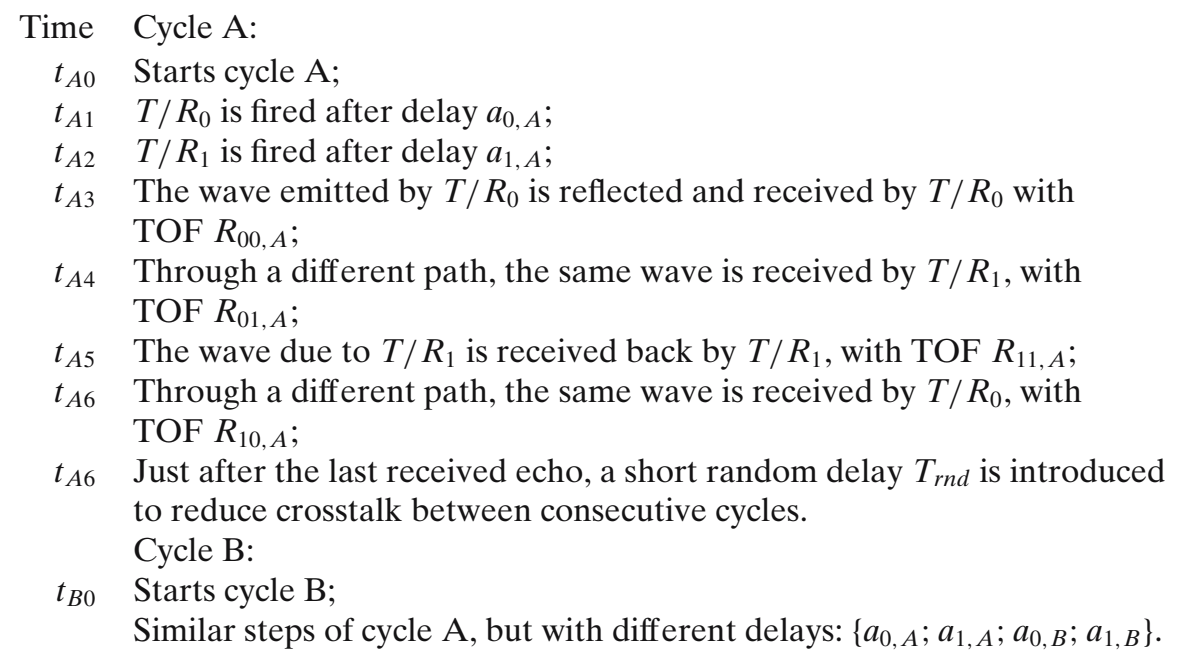

At the end of the second cycle, each sensor has collected four TOFs. By checking these consecutive sonar readings ( $R_{k j, l}$ values) and allowing only small differences, i.e. "near identical readings" it is possible to eliminate random errors, corrupted TOFs, and identify the important crossed echoes. Considering a Gaussian distribution for all measurements, the consecutive readings decision rule, for a two-tailed-test with a significance level of $5 \%{ }^{2}$ is presented in Table 2.

\footnotetext{
${ }^{2}$ From Eq. 3 , in the main lobe, $\sigma_{r}=2 \mathrm{~mm}$ corresponds to a traveling time, $\sigma_{T O F}$, of $11.6 \mu \mathrm{s}$ (for the speed of sound $c=344 \mathrm{~m} / \mathrm{s}$ ). A two-tailed significance level of $5 \%$ is bounded approximately by the critical values $\pm 2 \sigma_{T O F}$, resulting on $95 \%$ acceptance region of $4 \sigma_{T O F}=\Delta t=50 \mu \mathrm{s}$.
} 


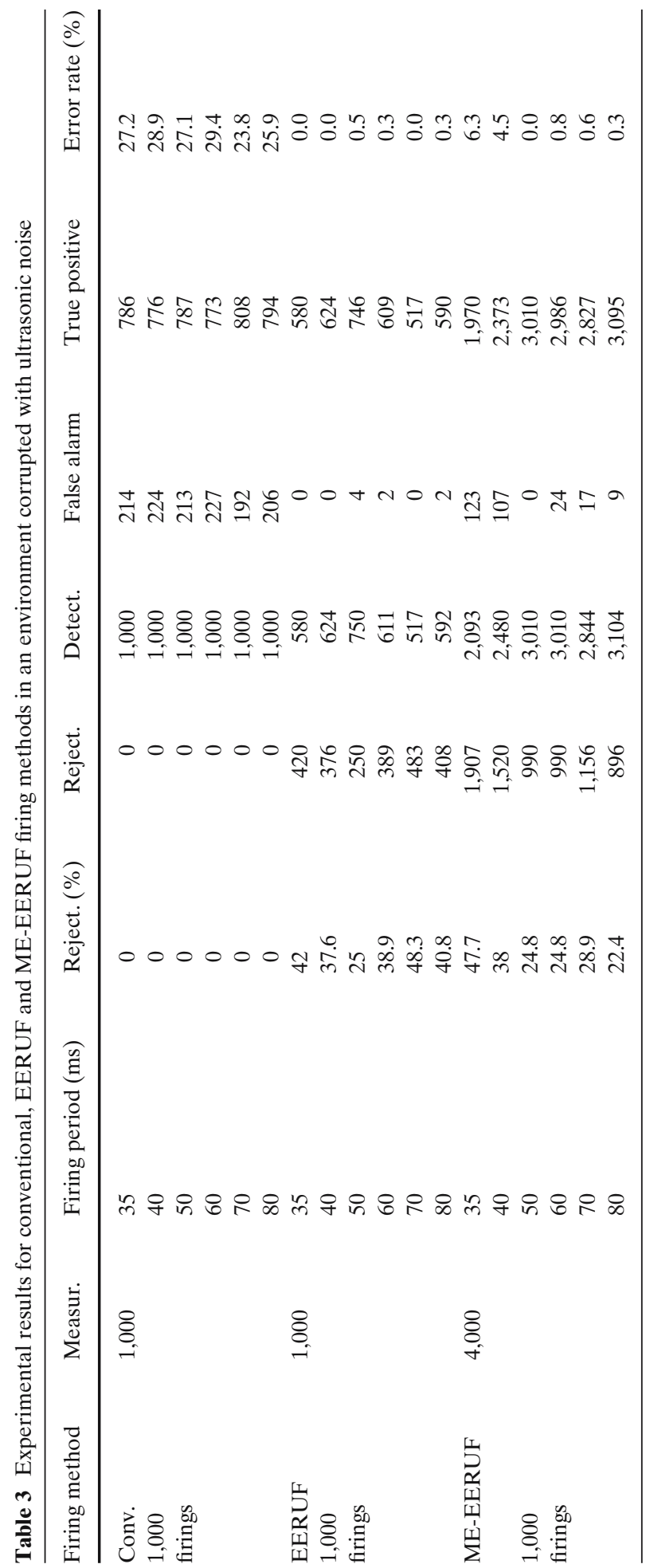




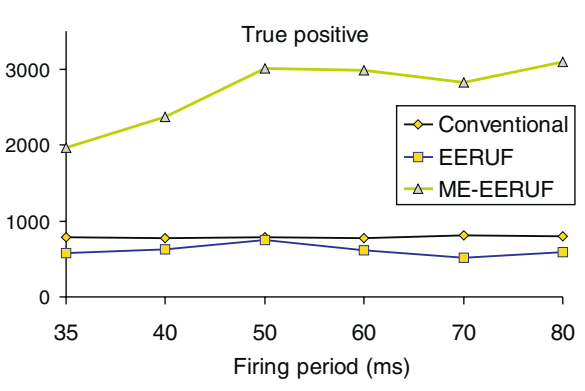

a

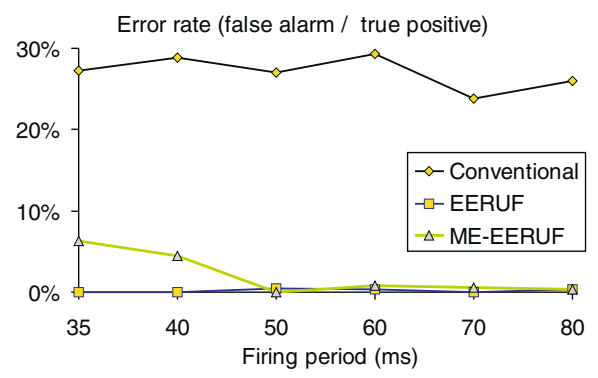

b

Fig. 8 a Total of true positive measurements; b error rate, ratio between false alarm and true positive measurements

\subsection{ME-EERUF Performance Analysis}

To test the ME-EERUF effectiveness, a simple experience was performed with the binaural system fired with three different modalities:

1. The conventional one, with independent sequential firings;

2. The EERUF modality, sequential with programmed alternate delays;

3. The ME-EERUF scheme, almost simultaneous fires with short alternate delays and multi-echo receiving functionality.

The system was tested acquiring 1,000 TOFs from a $1 \mathrm{~m}$ distance wall, using different firing periods, with an external interfering ultrasound transducer, firing straightly to the system at a $10 \mathrm{~Hz}$ rate. The results are presented in Table 3, and Fig. 8a,b.

With acquisition rates four to six times greater, the ME-EERUF shows high interference rejection capability, preserving a very low level of errors. Experimental results have shown successful rejection of undesired crosstalk and good acceptance of true measurements. With just two firing cycles, this system provides fast and more

Table 4 Precision and field-of-view, or orientation uncertainty, for the measurements $\mathbf{x}=\left[R_{0}, R_{1}, R_{2}\right]$, gathered from scanning the basic reflectors

\begin{tabular}{llll}
\hline Feature & Measurement & $\begin{array}{l}\text { Field of view } \\
\text { (degrees) }\end{array}$ & $\begin{array}{l}\text { Max. Std. } \\
(\mathrm{mm})\end{array}$ \\
\hline \multirow{2}{*}{ Wall } & $R_{0}$ & $-12.6+13.5$ & 1 \\
& $R_{1}$ & $-13.5+13.5$ & 1 \\
& $R_{2}$ & $-13.5+13.5$ & 3 \\
& visual-field & $-12.6+13.5$ & \\
Corner & $R_{0}$ & $-11.7+9.9$ & 2 \\
& $R_{1}$ & $-8.1+10.8$ & 2 \\
& $R_{2}$ & $-10.8+10.8$ & 3 \\
& visual-field & $-8.1+9.9$ & \\
& $R_{0}$ & $-8.1+8.1$ & 1.5 \\
& $R_{1}$ & $-9.9+5.4$ & 1.5 \\
& $R_{2}$ & $-6.3+8.1$ & 3 \\
& visual-field & $-6.3+5.4$ & \\
\hline
\end{tabular}


Fig. 9 Binaural system scanning a corner $\left(R_{2 C}\right)$

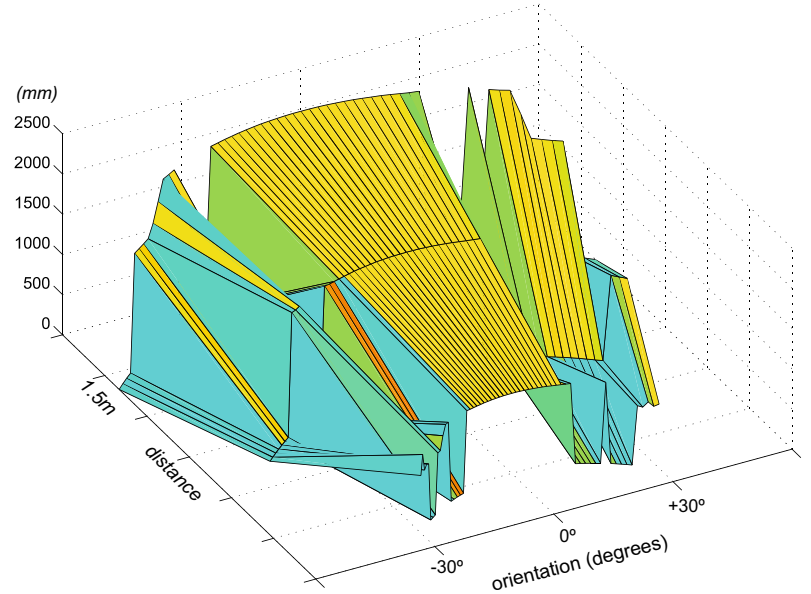

reliable information, leading to a higher obstacle detection probability. These results are important to implement feature detection and identification algorithms.

\subsection{Experimental System Modelling}

Work was carried out with the purpose of obtaining experimental models for the binaural system in the presence of walls, corners and edges. A rotating system with an angular resolution of $0.9^{\circ}$ was used to perform the environment scanning (one feature at a time) using the ME-EERUF firing method. A set of data was collected for the ranges of $0.5,1$ and $1.5 \mathrm{~m}$. As an example, Fig. 9 depicts an experimental view for the binaural system scanning a corner. As can be observed in the Figs. 3 and 9, lateral lobes information exhibits an higher variability and lower precision. Evaluated by geometry Eqs. 7, 9 and 11, the desired field-of-view can be bounded by central main perception lobe with low $\sigma_{C}$. These results are summarized in Table 4.

\section{Reflectors Recognition}

The purpose of this section is to present experimental results concerning reflector recognition (classification, localization, and orientation) for feature map set up, using the ME-EERUF method. Feature map is carried out by Bayesian classifiers, using the probabilistic information about the three classes of features, $\Omega=$ $\left\{\omega_{1}, \omega_{2}, \omega_{3}\right\}=\{$ wall, edge, corner $\}$. Figure 10 shows the overall recognition system, which will also be described in the following subsections. The data acquisition and the pre-processing blocks were already described in Section 3. Figure 11 shows the non-structured environment composed by several objects of different textures and materials such as paper box, concrete walls, wood, iron tubes, etc. This environment was used to test the overall recognition system depicted in Fig. 10. The embedded ultrasonic processing system (Fig. 11) includes a Microchip microcontroller running at $40 \mathrm{MHz}$, with capability to implement the firing schedule (block B), the filtering (block C), and the normalization (block D), of the classifier presented in Fig. 10. The 
Fig. 10 Block diagram of the classifier: $\mathbf{a}$ - natural patterns are captured from a non-structured laboratory; $\mathbf{b}$ - data is acquired by the ME-EERUF system; c - data is filtered; d - pattern tri-dimensional space is reduced to an unidimensional space; $\mathbf{e}-$ reflectors classification

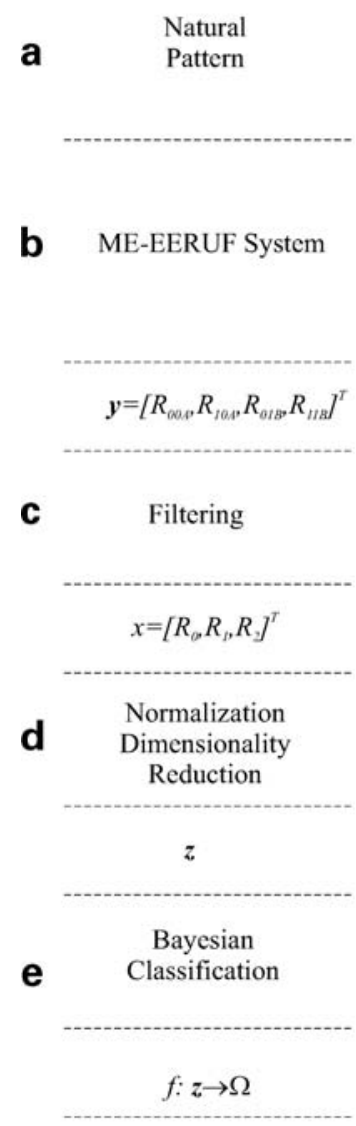

Reflectors

$\Omega=\left\{\omega_{l}, \omega_{2}, \omega_{3}\right\}$

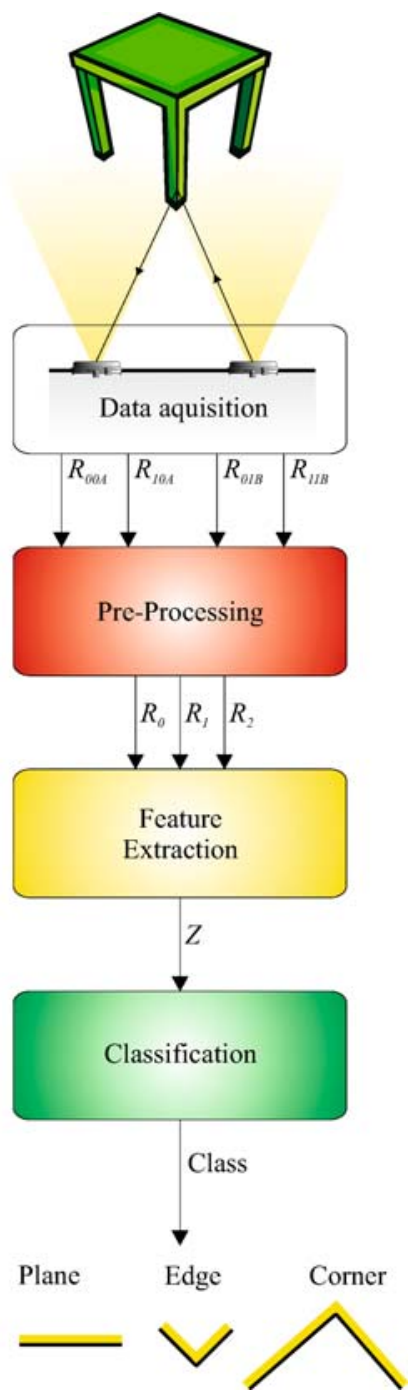

processed result $z_{E}(\mathbf{x})$ (Eq. 12) is sent through a CAN bus to an higher level PCbased processing system (block E), which performs real-time feature classification. The prototype depicted in Fig. 11 is placed on the top of a rotating system at a height of $60 \mathrm{~cm}$. Acquisition of 15 samples was carried out at every angular displacement of $0.9^{\circ}$.

\subsection{Feature Extraction}

Given a set of measurements, $\mathbf{x}$, from the ME-EERUF system, the problem is to decide the class $\omega$ to which this new pattern belongs (Fig. 10). This statistical pattern recognition problem is well solved by using the Bayes's rule [5], however it needs the class conditional densities $p\left(z \mid \omega_{i}\right)$, and their a priori probabilities $P\left(\omega_{i}\right)$. 


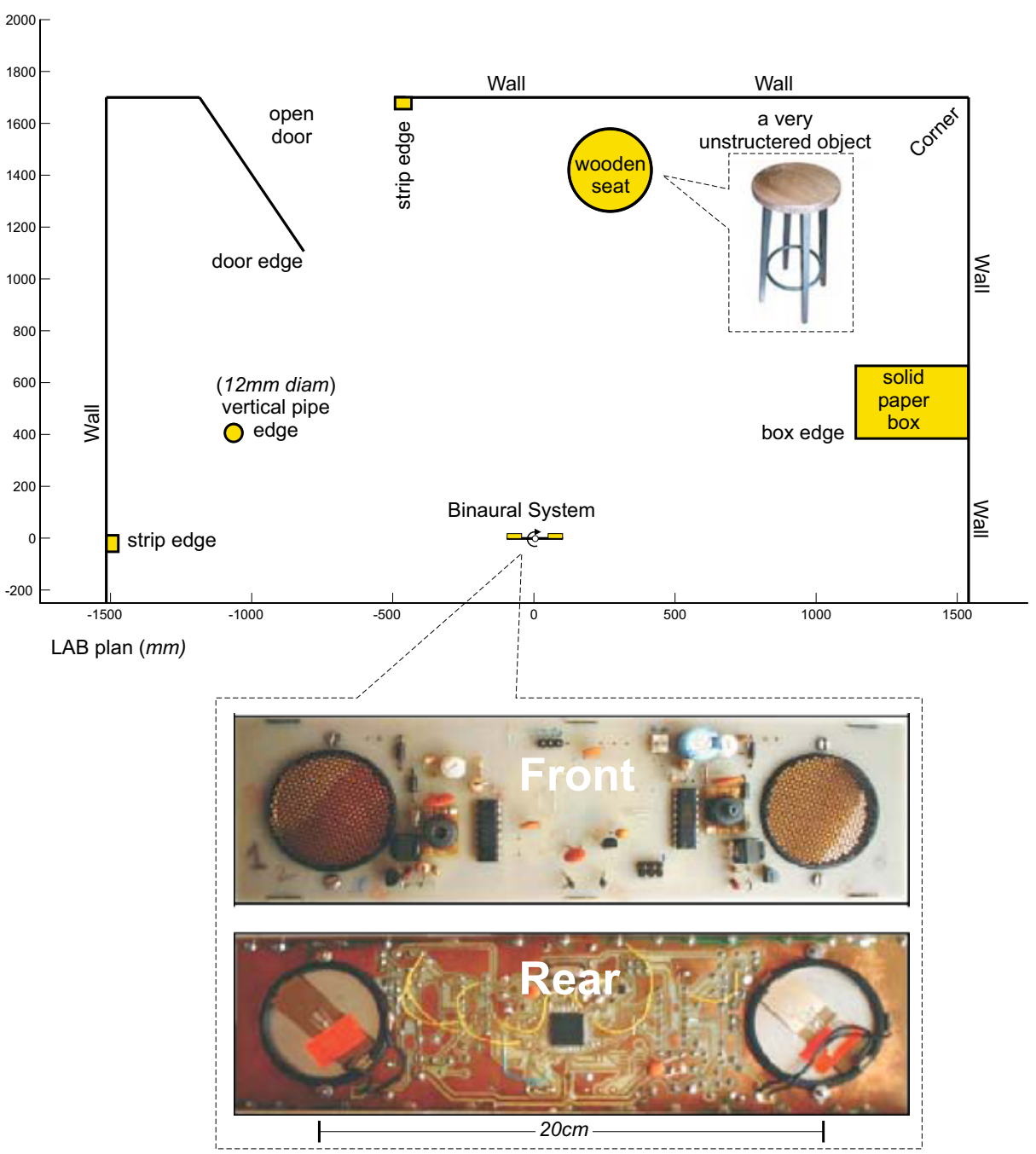

Fig. 11 Non-structured environment used in the experiments, including the front and rear view of a prototype of the binaural system

Combining and transforming the three observed measurements, it is possible to reduce the pattern dimensional space, simplifying and improving the performance of the classifier. Considering $D=\left(\frac{d}{2}\right)^{2}$ and $K=\left(\frac{R_{1}-R_{0}}{2}\right)^{2}$, the following normalized relations can be obtained manipulating Eqs. 6, 8 and 10:

$$
\begin{aligned}
& z_{E}(\mathbf{x})=\frac{R_{2}{ }^{2}-\left(\frac{R_{0}+R_{1}}{2}\right)^{2}}{K-D} \\
& z_{W}(\mathbf{x})=z_{E}(\mathbf{x})+1 \\
& z_{C}(\mathbf{x})=z_{E}(\mathbf{x})-1
\end{aligned}
$$


Considering $z_{E}$ as the input, a set of unidimensional training patterns was obtained for each reflector at different distances and orientations (see Fig. 12). This set of training data was used to estimate the class conditional densities, whose expectation, $\mu_{i}$, and standard deviation, $\sigma_{i}$, are expressed as follows:

$$
\begin{aligned}
p\left(z \mid \omega_{i}\right) & \approx N\left(\mu_{i}, \sigma_{i}\right) \\
p(z \mid \text { Wall }) & \approx N(-1,0.27) \\
p(z \mid \text { Edge }) & \approx N(0,0.44) \\
p(z \mid \text { Corner }) & \approx N(1.6,0.7)
\end{aligned}
$$

and the a priori probabilities are estimated by empirical frequencies of the training set

$$
P\left(\omega_{i}\right)=\frac{\# \text { samples in class } \omega_{i}}{\text { Total \# of samples }}
$$

It is worth noting that the experimental model for the corner (Eq. 15) is slightly different from the theoretical one (Eq. 14), i.e. the expectation is 1.6 while in the theoretical model is 1 . This can be explained as follow: while $\left[R_{0}, R_{1}, R_{2}\right]$ are equally affected by attenuation due to a single reflection for wall and edge reflectors (see Fig. 6), $R_{2}$ is affected by two reflections in the corner case, increasing the $R_{2}$ value and therefore $\mu_{C}$.

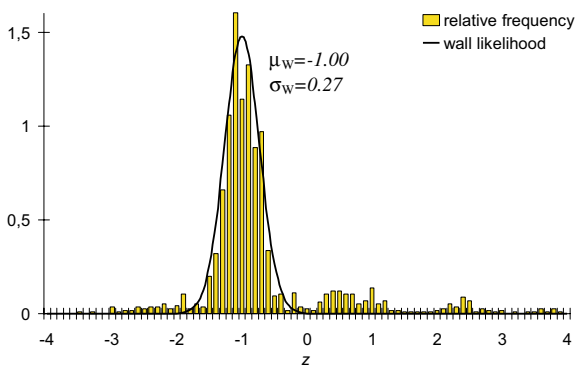

a

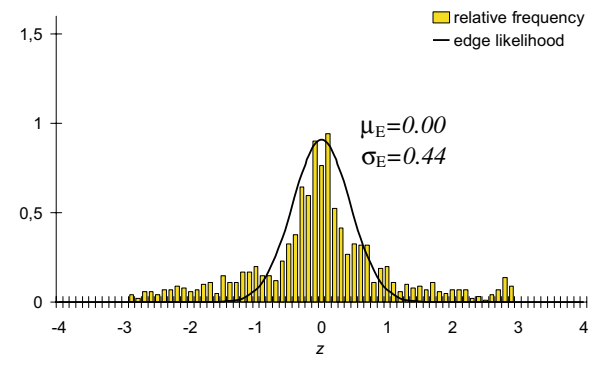

b

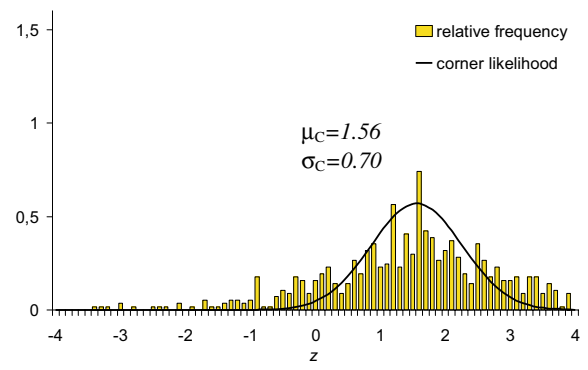

C

Fig. 12 Training data. A total of 3,600 acquisitions for each reflector at different ranges $(0.5,1,1.5 \mathrm{~m})$ and different orientations $\left(-30\right.$ to $\left.+30^{\circ}\right)$ : a training set resulting from wall reflector; $\mathbf{b}$ training set resulting from edge reflector; $\mathbf{c}$ training set resulting from corner reflector 


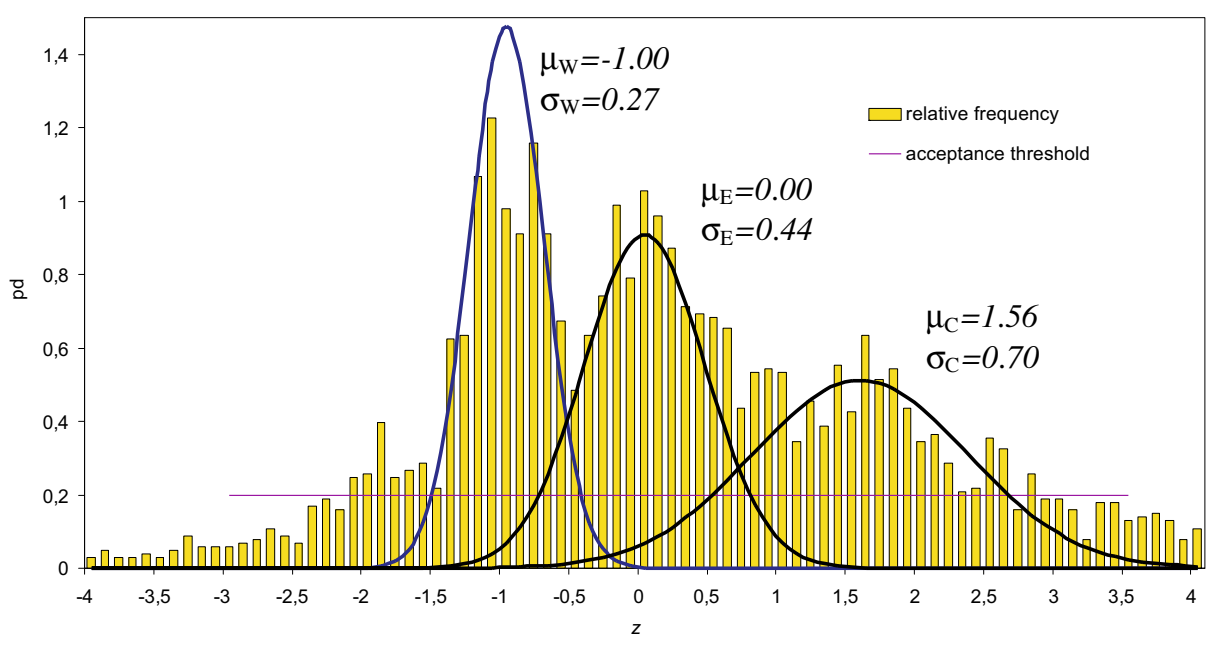

Fig. 13 Training data. Acquisitions were obtained for the non-structured environment of Fig. 11

In order to analyze the robustness of the training set parameters, a new set of unidimensional training patterns was obtained, for the non-structured environment presented in Fig. 13, at different distances and orientations. The results show that the conditional densities estimated for each reflector (see Fig. 12) are in accordance with the results presented for the non-structured environment. For this classification system there are three distributions $p\left(z \mid \omega_{1}\right), p\left(z \mid \omega_{2}\right), p\left(z \mid \omega_{3}\right)$ (see Fig. 12), which overlap, and thus have non-zero Bayes classification error [5]. Any pattern from $\omega_{2}$ could be properly classified as $\omega_{2}$ (a "hit"), or misclassified as $\omega_{1}$ (a "false alarm").

\subsection{Classification}

To design a Bayesian classifier, the expectation $\mu_{i}$, and the standard deviation $\sigma_{i}$, for the three classes (wall, edge, and corner), defined by Eq. 15 are considered. The idea is to use the expectation of each class and the respective standard deviation, directly as generative models for the trials [11]. Assuming a Gaussian distribution, the probability of observing a class $\omega_{i}$ for a given measurement $z_{i}$, can be expressed by the conditional density function:

$$
p\left(z \mid \omega_{i}\right)=\frac{1}{\sqrt{2 \pi} \sigma_{i}} \exp \left(-\frac{\left(z-\mu_{i}\right)^{2}}{2 \sigma_{i}^{2}}\right)
$$

The a priori probabilities, and conditional densities are sufficient to design optimal classifiers. However, another probability has to be previously derived: the a posteriori probability $P\left(\omega_{i} \mid z\right)$. It is the probability that an object belongs to class $\omega_{i}$ given that the measurement associated with the object is $z$. According to the Bayes theorem for conditional probabilities:

$$
P\left(\omega_{i} \mid z\right)=\frac{p\left(z \mid \omega_{i}\right) p\left(\omega_{i}\right)}{\sum_{j=1}^{3} p\left(z \mid \omega_{j}\right) P\left(\omega_{j}\right)}
$$


Table 5 Cost function for wall, edge, and corner classification

\begin{tabular}{lcrrr}
\hline$C\left(\hat{\omega}_{k} \mid \omega_{i}\right)$ & & \multicolumn{2}{l}{ True class } & \\
\cline { 5 - 5 } \cline { 5 - 5 } Assigned class & & Wall & Edge & Corner \\
\hline Wall & -1 & 0.5 & 0.8 \\
Edge & 0.5 & -0.9 & 0.2 \\
Corner & 0.8 & 0.2 & -0.7 \\
\hline
\end{tabular}

Based on this, three decision rules were then derived:

- minimum risk (MR) classifier;

- maximum a posteriori probability classifier (MAP) (uniform cost function);

- minimum distance (MD) classifier.

\subsubsection{Minimum Risk Classifier}

If an arbitrary classifier assigns a class $\hat{\omega}_{k}$ to a measurement $z$ coming from an object with true class $\omega_{i}$, then a cost $C\left(\hat{\omega}_{k} \mid \omega_{i}\right)$ is involved. The a posteriori probability of having such an object is $P\left(\omega_{i} \mid z\right)$. Therefore, the expectation of the cost is [8]:

$$
R\left(\hat{\omega}_{k} \mid z\right)=E\left[C\left(\hat{\omega}_{k} \mid \omega_{i}\right)\right]=\sum_{i=1}^{N} C\left(\hat{\omega}_{k} \mid \omega_{i}\right) P\left(\omega_{i} \mid z\right)
$$

The quantity $R\left(\hat{\omega}_{k} \mid z\right)$ is called the conditional risk. The optimal classifier is the one with minimum risk. Therefore, the Bayes classifier takes the form:

$$
\hat{\omega}_{B A Y E S}=\hat{\omega}_{i}: R\left(\hat{\omega}_{i} \mid z\right) \leq R\left(\hat{\omega}_{j} \mid z\right), \forall j \neq i
$$

Table 5 shows the assigned cost function for the MR classifier.

\begin{tabular}{|c|c|c|c|c|c|c|c|}
\hline & \multirow[t]{3}{*}{ Assigned class } & \multicolumn{6}{|c|}{ True class } \\
\hline & & \multicolumn{3}{|l|}{ "Hit" } & \multicolumn{3}{|c|}{ "False alarm" } \\
\hline & & Wall & Edge & Corner & Wall & Edge & Corner \\
\hline \multirow[t]{3}{*}{ MAP } & Wall & 17 & - & - & - & 7 & 1 \\
\hline & Edge & - & 28 & - & 0 & - & 1 \\
\hline & Corner & - & - & 11 & 4 & 10 & - \\
\hline \multirow[t]{3}{*}{ MR } & Wall & 16 & - & - & - & 7 & 1 \\
\hline & Edge & - & 29 & - & 0 & - & 0 \\
\hline & Corner & - & - & 12 & 4 & 9 & - \\
\hline \multirow[t]{3}{*}{ MD } & Wall & 14 & - & - & - & 6 & 0 \\
\hline & Edge & - & 35 & - & 0 & - & 0 \\
\hline & Corner & - & - & 13 & 8 & 4 & - \\
\hline
\end{tabular}

Table 6 Classification performance - pairwise comparison 

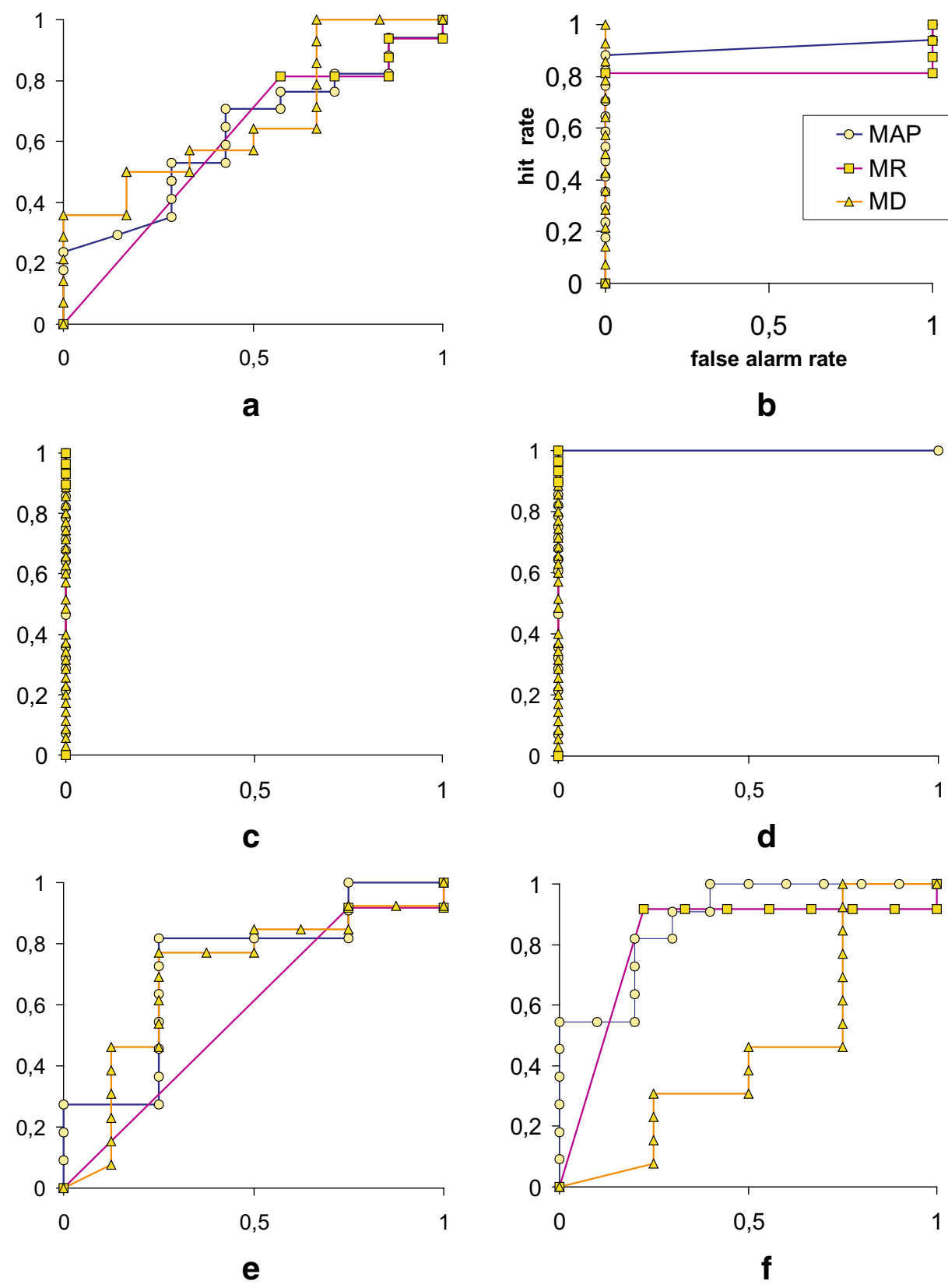

Fig. 14 "Hit" rate versus "false alarm" rate pairwise comparison: a wall-edge ROC; b wall-corner ROC; $\mathbf{c}$ edge-wall ROC; d edge-corner ROC; e corner-wall ROC; f corner-edge ROC 


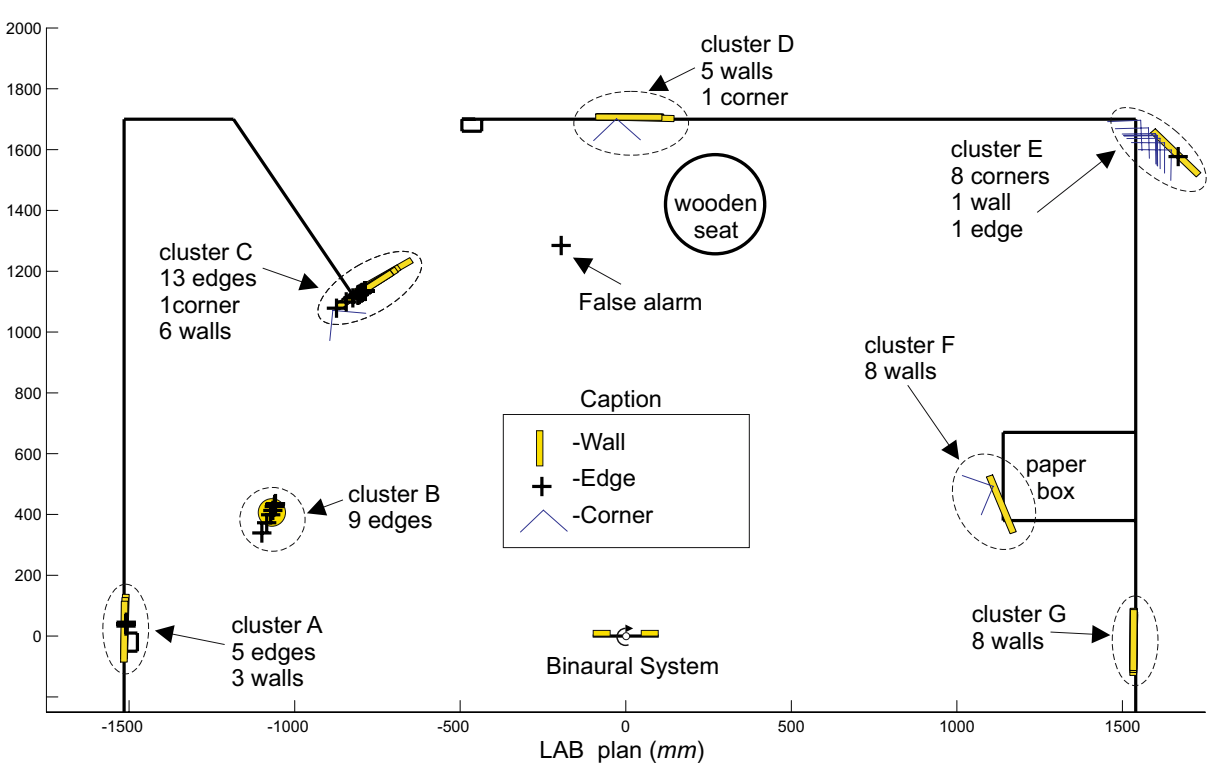

Fig. 15 Results for the MAP classifier

\subsubsection{MAP Classifier}

If a unit cost is assumed when an object is misclassified, an uniform cost function is obtained. When the classification is correct a zero cost function is obtained. In this case, the minimization of the risk is equivalent to the maximization of the a posteriori probability. Therefore, the decision rule takes the form [8]:

$$
\hat{\omega}_{B A Y E S}=\operatorname{argmax}_{i=1 \ldots 3}\left(P\left(\omega_{i} \mid z\right)\right), \forall \omega \in \Omega
$$

Application of Bayes' theorem for conditional probabilities and cancelation of irrelevant terms yields a classification equivalent to a MAP classification, but expressed in terms of the a priori probabilities, and the conditional probability densities:

$$
\hat{\omega}_{B A Y E S}=\operatorname{argmax}_{i=1 \ldots 3}\left(p\left(z \mid \omega_{i}\right) P\left(\omega_{i}\right)\right), \forall \omega \in \Omega
$$

\subsubsection{Minimum Distance Classifier}

To obtain the linear classifier a further development of Bayes classification with uniform cost function is required. Substitution of Eq. 17 in Eq. 22 gives the following classification [8]:

$$
\begin{aligned}
\hat{\omega}(z) & =\omega_{k} \\
k & =\operatorname{argmax}_{i=1 \ldots 3}\left\{\frac{1}{\sqrt{2 \pi} \sigma_{i}} \exp \left(-\frac{\left(z-\mu_{i}\right)^{2}}{2 \sigma_{i}^{2}}\right) P\left(\omega_{i}\right)\right\}
\end{aligned}
$$




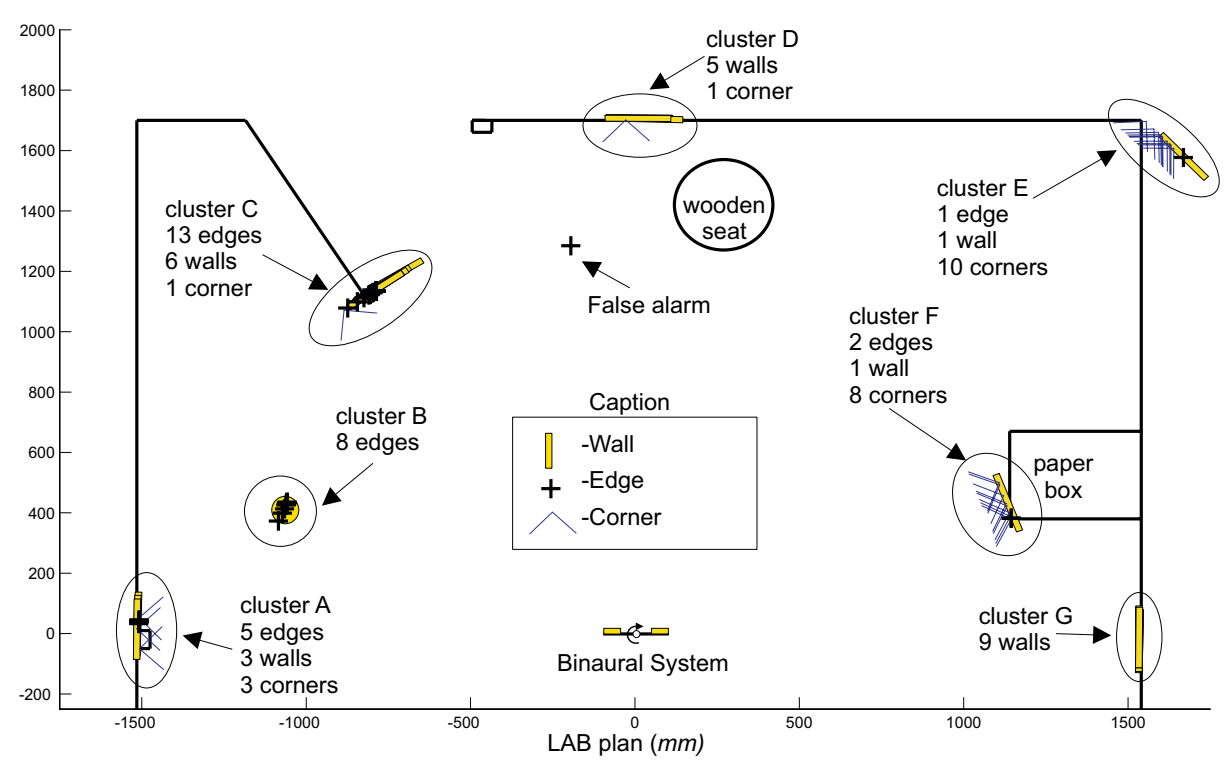

Fig. 16 Results for the MR classifier

Taking the logarithm of the function between brackets, the following decision rule is obtained:

$$
\begin{aligned}
& \hat{\omega}(z)=\omega_{k} \\
& k \quad=\operatorname{argmax}_{i=1 \ldots 3}\left\{-\ln \left(\sigma_{i}\right)+2 \ln P\left(\omega_{i}\right)-\frac{\left(z-\mu_{i}\right)^{2}}{\sigma_{i}}\right\}
\end{aligned}
$$

Considering that, for the given data set the standard deviation is very similar, $\sigma_{\text {Wall }}, \sigma_{E d g e}$, and $\sigma_{\text {Corner }}$ are replaced by a single constant $\sigma$, and the decision rule is changed to:

$$
\begin{aligned}
& \hat{\omega}(z)=\omega_{k} \\
& k \quad=\operatorname{argmax}_{i=1 \ldots 3}\left\{2 \ln P\left(\omega_{i}\right)-\frac{\left(z-\mu_{i}\right)^{2}}{\sigma}\right\}
\end{aligned}
$$

The quantity $\left(z-\mu_{i}\right)^{2}$ can be regarded as a distance measurement between $z$ and the expectation value $\mu_{i}$. The function (25) decides in favour of the class whose expectation is nearest to the observed measurement [8].

\subsection{Feature Map Results}

Receiver operating characteristic (ROC) analysis is a widely used method for analyzing the performance of two-class classifiers. Since the present case is a three-class classifier, a pairwise ROC analysis was carried out. Pairwise comparisons break down a N-class classification problem into separate binary one-versus-one comparisons. For a $\mathrm{N}$-class classification, there are $N(N-1)$ different binary comparisons. Thus, this method returns $N(N-1)$ pairwise ROC curves for each paired comparison. 


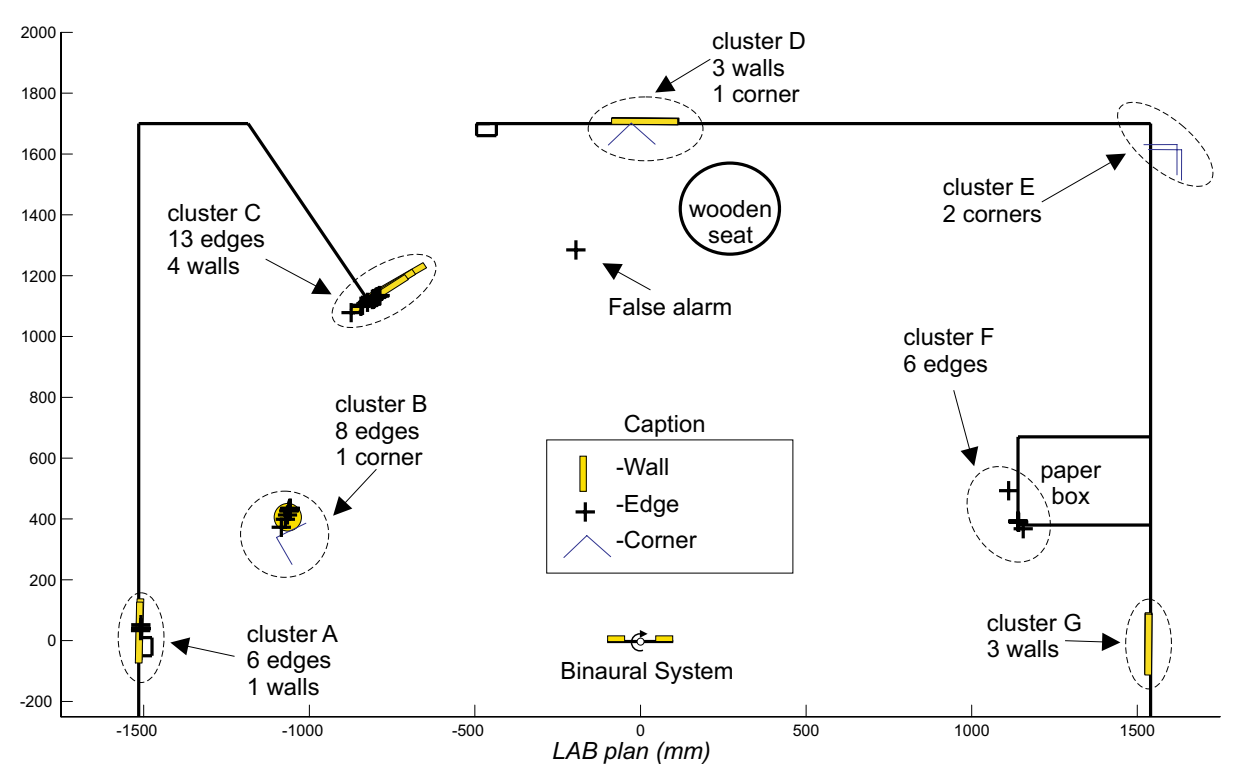

Fig. 17 Results for the MD classifier

The three classifiers presented in Section 4.2 were applied to the unidimensional normalized data, $z$, which resulted from the application of the acquisition and preprocessing systems (see Fig. 10) to the non-structured environment depicted in Fig. 11. Bearing in mind the three classes in analysis, Table 6 shows the number of "hits" and "false alarms" for the three classifiers. Table 6 shows that the MD classifier presents the lowest number of "false alarms" for all classes except the pair corner-wall. The MD classifier also shows the higher number of "hits" for edges and corners, but it presents the lowest number of "hits" for walls. The MR and the MAP classifier present very similar results. Figure 14 shows the ROC curves resulting from the pairwise comparison presented in Table 6.

The area under the ROC curve (AUC) is a numeric performance metric, which represents how separable two objects are. An AUC of 1 suggests that the classifier would always be able to distinguish a "hit" from a "false alarm." A straight line corresponding to all the points at which the "hit" rate and the "false alarm" rate are equal to each other indicates chance classification and this has an AUC of 0.5. Chance classification means that when posed with the task of distinguishing a "hit" from a "false alarm," the classifier could at best "guess" to which class the object belongs. If the AUC is less than 0.5 most of the classification are incorrect. If all the results are "hits," the "false alarm" rate is always zero, and the ROC is a vertical line in the $y$-axis.

The results presented in Fig. 14 show that the performance of the classifiers show several similarities. The corner-edge ROC (Fig. 14f) shows that the MD classifier has low performance, even though there were only four "false alarms" for this case (see Table 6). This situation occurs because the misclassified corners do not disappear even when the threshold is close to the limit. The opposite situation occurs for the corner-wall ROC. In this case the MD classifier presents the highest level of "false 
alarms", but the ROC curve shows a reasonable performance. The classifiers show a better performance between classes with conditional density functions that do not overlap so much. For instance, the wall-corner performance is significantly higher than the wall-edge.

Figures 15, 16, and 17 present the classification results for the non-structured environment depicted in Fig. 11. In this case a threshold was applied in order to reduce misclassifications. The application of the threshold also eliminates some good classification results, and because of this, its choice must be taken carefully. Since most of good classifications belong to a cluster corresponding to the same object (because data is obtained every $0.9^{\circ}$ ), the elimination of some "hits" may not be a problem. This cluster analysis will be one of the subjects of future work. The analysis of Figs. 15, 16, and 17, show that all classifiers were able to identify most of the reflectors "visible" according to Table 4. Let us consider cluster E for illustrating the interpretation of results shown in Fig. 15. What can be observed in this case, is that for the same scan the corner (true class) was classified eight times as corner, one time as wall and one time as edge. A singular case occurs for the wooden seat, for which none reflectors were identified, because it is a highly unstructured object.

\section{Conclusions}

The new firing technique (ME-EERUF) allows high data acquisition rates with a high level of error rejection (see Fig. 8). This technique can be easily implemented with simple sensor configurations, and provides essential information for reflector recognition. The results obtained using three Bayes' classifiers, show the potentiality of the method. An unstructured environment, a conventional laboratory room represented in Fig. 11, was used to test the three classifiers: MAP, MR, and MD. The MD classifier presents the lowest level of "false alarms." The MAP and the MR classifier present similar results. However, the need to establish a cost matrix for the MR classifier may be difficult to define.

These classification methods will be applied in robotic navigation, using time integration and fusion with other sensor classification modules. These results are the first step in the global development that will integrate several combined binaural cells, vision, laser range-finder, and RADAR, in order to have a complete perception of the environment. Several configuration networks integrating different sensors offboard and on-board will be tested.

The binaural system, in particular, is also being improved. New functionalities and methodologies will be applied, such as: signal amplitude, phase shift measurement, burst codification, and more embedded processing capabilities.

This system is being developed considering its application in the RobChair sonar system [15], which is a project aiming to assist physically and mentally handicapped persons in steering and controlling powered intelligent wheelchairs.

Acknowledgements This work was partially supported by FCT (Portuguese Foundation for Science and Technology) under Grant: POSC/EEA-SRI/58279/2004. Fernando Moita is with the Department of Electrical Engineering, Polytechnic Institute of Coimbra and acknowledges a research fellowship from FCT: SFRH/BD/27771/2006. Ana C. Lopes is with the Department of Electrical Engineering, Polytechnic Institute of Tomar and acknowledges a research fellowship from FCT: SFRH/BD/29655/2006. 


\section{References}

1. Araujo, E.G., Grupen, R.A.: Feature extraction for autonomous navigation using an active sonar head. In: IEEE International Conference on Robotics and Automation (ICRA'2000), pp. 3823$3828(2000)$

2. Barshan, B., Kuc, R.: Differentiating sonar reflections from corners and planes by employing an intelligent sensor. IEEE Trans. Pattern Anal. Mach. Intell. 12(6), 560-569 (1990)

3. Borenstein, J.: The vector field histogram - fast obstacle avoidance for mobile robots. IEEE Trans. Robot. Autom. 7(3), 278-288 (1991)

4. Borenstein, J., Koren, Y.: Error eliminating rapid ultrasonic firing for mobile robot obstacle avoidance. IEEE Trans. Robot. Autom. 11, 132-138 (1995)

5. Duda, R.O., Hart, P.E.: Pattern Classification and Scene Analysis. Wiley-Interscience, Statford Research Institute, Menlo Park, California (1973)

6. Elfes, A.: Occupancy grids: a stochastic spatial representation for active robot perception. In: 6th Conference on Uncertainty in AI (1990)

7. Harris, K.D., Recce, M.: Experimental modelling of time-of-flight sonar. Robot. Auton. Syst. 24, 33-42 (1998)

8. van der Heijden, F., Duin, R., de Ridder, D., Tax, D.: Classification, Parameter Estimation and State Estimation - an Engineering Approach Using Matlab. John Wiley \& Sons, England (2004)

9. Hernández, Á., Ureña, J., García, J.J., Mazo, M., Hernanz, D., Dérutin, J.P., Sérot, J.: Ultrasonic ranging sensor using simultaneous emissions from different transducers. IEEE Trans. Ultrason. Ferroelectr. Freq. Control 51(12), 1660-1670 (2004)

10. Kleeman, L., Kuc, R.: Mobile robot sonar for target localization and classification. Int. J. Rob. Res. 14, 295-318 (1995)

11. Kohlmorgen, J., Blankertz, B.: Bayesian classification of single-trial event-related potentials in EEG. Int. J. Bif. Chaos 14(2), 719-726 (2004)

12. Kuc, R., Siegel, M.: Physically based simulation model for acoustic sensor robot navigation. IEEE Trans. Pattern Anal. Mach. Intell. 9, 766-778 (1987)

13. Moita, F., Nunes, U.: Ultrasonic reflectors recognition with a fast firing system. In: SICICA 2003 5th IFAC International Symposium on Intelligent Components and Instruments for Control Applications. Aveiro, Portugal (2003)

14. Moita, F., Feijão, A., Nunes, U., de Almeida, A.T.: Modelling and calibration of an ultrasonic ranging system of a mobile robot. In: Controlo 94. Lisbon (1994)

15. Pires, G., Nunes, U.: A wheelchair steered through voice commands and assisted by a reactive fuzzy-logic controller. J. Intell. Robot. Syst. 34, 301-314 (2002)

16. Pires, G., Martins, N., Almeida, R., Moita, F., Nunes, U., de Almeida, A.T.: Optimization of the tri-aural system, detection and identification of different reflectors. In: Mechatronics 96 with M $^{2}$ VIP 96 Conference. Guimarães, Portugal (1996)

17. Randall, R.C., Michel, H.E.: Theoretical design issues of a multi-sensor doppler-tolerant ultrasonic system using pseudorandom codes. In: The 2006 World Congress in Computer Science, Computer Engineering, and Applied Computing (ICAI'06), pp. 628-634. Las Vegas, Nevada, USA (2006)

18. Sabatini, A.M.: Active hearing for external imaging based on an ultrasonic transducer array. In: IEEE/RSJ International Conference on Intelligent Robotic Systems (IROS'92), pp. 829-836 (1992)

19. Simmons, J.A., Ferragamo, M.J., Moss, C.F.: Echo-delay resolution in sonar images of the big brown bat, Eptesicus fuscus. In: National Academy of Science, vol. 95, pp. 12,647-12,652 (1998)

20. Wijk, O., Christensen, H.: Triangulation-based fusion of sonar data with applications in robot pose tracking. IEEE Trans. Robot. Autom. 16, 740-752 (2000) 